\title{
与肿瘤转移相关的 $\mathrm{N}$ 型胶原酶单链抗体的研制
}

侵袭和转移是恶性肿瘤的重要特征. IV 型胶原酶在肿瘤组织中的过度表达和活性增 高被认为与肿瘤侵袭和转移密切相关 ${ }^{[1]}$.

许多实验表明, 具有抑制 IV 型胶原酶活性 的物质(如金属蛋白酶抑制剂,特异抗体)均能 够有效地抑制或降低肿瘤的转移. 因此, 通过 IV 型胶原酶抑制物来阻止肿瘤转移已成为肿 瘤治疗研究的策略之一。 IV 型胶原酶抗体以 其特异性和中和作用, 可能阻断肿瘤的侵袭和 转移 ${ }^{[2]}$, 显示用于肿瘤免疫治疗的前景.

本研究是利用噬菌体抗体展示技术 ${ }^{[3]}$, 研制出 IV 型胶原酶特异单链抗体. 该抗体仅 含有抗体的轻链和重链可变区, 分子量为 27 $\mathrm{ku}$, 是完整抗体分子量的六分之一．我们采用 的技术方案是: 从分泌 $N$ 型胶原酶单克隆抗体 的杂交瘤细胞 3D6 中提取并纯化 mRNA. 体 外反转录 cDNA. 用 PCR 技术分别获得抗体 轻链可变区 $\left(V_{L}\right)$ 和重链可变区 $\left(V_{H}\right)$ 基因, 用 编码 $\left(\mathrm{Gly}_{4} \mathrm{Ser}\right)_{3}$ 的连接 DNA 把轻链和重链可 变区基因连接起来, 形成 $\mathrm{V}_{\mathrm{H}}$-linker- $\mathrm{V}_{\mathrm{L}}$ 单链抗 体基因. 以单链抗体基因为模板, 再进行 PCR 扩增, 扩增产物经琼脂糖凝胶电泳分离 后, 用 Sephaglas ${ }^{\mathrm{TM}}$ BandPrep Kit 回收单链抗 体基因. 随后用限制性内切酶 Sfi 1 和 Not 1 分别消化单链抗体基因, 使其形成粘性末端, 与经过 Sfi 1 和 Not 1 双酶切的 pCANTAB5E 载体连接. 将含单链抗体基因的重组质粒转 化大肠杆菌 TG1 感受态细胞. 在辅助噬菌体 KO7 辅助下, 产生重组噬菌体抗体. 经过 3 轮免疫亲和篮选和 ELISA 检测, 我们把与 IV 型胶原酶结合活性最高的阳性克隆挑选出来, 命名为 COIV19.

为了获得可溶性 $\mathrm{N}$ 型胶原酶单链抗体, 我 们把 COIV-19 的基因转入大肠杆菌 HB2151.
转化子在 IPTG 的诱导下, 表达可溶性 IV 型胶 原酶单链抗体. 收集含单链抗体的培养上清, 并用 50 \% 硫酸铵沉淀浓缩, 经过 Sephadex G75 层析后, 获得纯度 $>80 \%$ 的 COIV-19 单链 抗体. 免疫印迹显示 COIV-19 分子量为 27 $\mathrm{ku}$. 免疫活性鉴定证明该单链抗体能够与 $\mathrm{N}$ 型胶原酶特异性结合, 不与人血清白蛋白发生 交叉反应.

抗体 COIV-19 是目前已知的唯一能够与 IN 型胶原酶特异结合的小分子基因工程抗体. 其特点是它保留了原单克隆抗体的特异性,而 分子量小. 因此它将可能作为肿瘤靶向载体, 携带抗肿瘤药物用于恶性肿瘤的免疫治疗. 目前将 COIV-19 与抗肿瘤抗生素 C1027 的基 因重组正在进行中.

致谢 本项工作为国家自然科学基金(批准号: 39470661)和卫生部科学研究基金资助项目.

\section{参考文 献}

1 Thompson R R, Iwamoto E W. Effects of inhibitors of plasminogen activator, serine proteinases, and collagenase IV on the invasion of basement membranes by metastatic cells. Cancer Research, 1988, 48: 3307

2 Hoythya M, Hujanen E. Modulation of type-IV collagenase activity and invasion behavior of metastatic human melanoma (A2058) cells in vitro by monoclonal antibodies to type-IV collagenase. Int J Cancer, 1990, 46: 282

3 McCafferty J, Griffiths A D, Winter G, et al. Phage antibodies: filamentous phase displaying antibody variable domains. nature, 1990, 348: 552

$$
\text { 田华松 }{ }^{(1)} \text { 阎锡蕴 }{ }^{(* *} \text { 江 敏 }{ }^{2}
$$
甄永苏象波 ${ }^{(1)}$

(1)中国科学院微生物研究所, 北京 100080; (2)中国医学科 学院医药生物技术研究所, 北京 100050 .*联系人) 